\title{
Descripción Anatómica de los Segmentos Óseos que Componen el Cráneo de la Especie Pudu puda
}

\author{
Anatomical Description of the Bone Segments that \\ make up the Skull of the Pudu puda Species
}

Saldivia, M. \& Villegas, F.

SALDIVIA, M. \& VILLEGAS, F. Descripción anatómica de los segmentos óseos que componen el cráneo de la especie Pudu puda. Int. J. Morphol., 37(1):167-173, 2019.

RESUMEN : El Pudú (Pudu puda), uno de los ciervos más pequeños del mundo y autóctono de Chile, es considerado como vulnerable (VU) en todo el territorio nacional, hay escasa información específica de los sistemas orgánicos que lo componen y cada vez es más común encontrarlos en zonas urbanas. Estas problemáticas hacen despertar la necesidad de fortalecer la investigación y el estudio de esta especie. En consecuencia, en el presente estudio se realizó una descripción anatómica del esqueleto craneal de dos ejemplares de Pudu puda, donde se destacaron sus principales características óseas comparándolas con especies domesticas ya estudiadas (ovino y caprino). Así se logró determinar diferencias entre las especies estudiadas y la necesidad de profundizar el análisis a través de mediciones de las piezas óseas.

PALABRAS CLAVE: Pudú; Descripción anatómica; Esqueleto craneal.

\section{INTRODUCCIÓN}

El Pudú es el más pequeño de los ciervos nativos de Chile (y de hecho uno de los ciervos más pequeños del mundo). Dentro del sector científico la especie recibe también el nombre de Pudu puda. Su distribución se da entre el Maule y Aysén, posiblemente en el norte de Magallanes y abundante en la isla de Chiloé (Chester, 2008).

Esta es una especie, con escasa información específica referente a los distintos sistemas orgánicos que la componen, y su presencia en las zonas urbanas es cada vez mayor, debido al creciente desarrollo de las ciudades. Esto genera, la necesidad de potenciar los conocimientos sobre la morfología de esta especie.

El presente estudio es el inicio de un extenso pero necesario trabajo que busca contribuir al desarrollo de la anatomía animal y tiene como objetivo realizar una descripción anatómica de los segmentos óseos que componen el cráneo de dos ejemplares (hembra y macho) de la especie pudú, guardando relación con los términos direccionales y planímetros expresados en la nómina anatómica veterinaria actual (International Committee on Veterinary Gross Anatomical Nomenclature, 2012) y considerando de forma esquemática las dos principales regiones de división, región craneana o neurocráneo y región facial o esplacnocráneo, así como cada subregión que las componen. Separando también en detalle las estructuras visibles en la superficie externa e interna de la cavidad craneana.

Los resultados de este estudio buscaron fundar una base sólida en el conocimiento de la arquitectura ósea y así contribuir y como una pieza fundamental en el desarrollo de la morfología en especies de fauna silvestre.

\section{MATERIAL Y METODO}

El material asociado a este estudio es de tipo biológico. En el cual se utilizaron los cráneos de dos ejemplares adultos (hembra y macho) de la especie Pudu puda, ambos obtenidos en años previos a este estudio a través de la técnica de osteotecnia en la unidad de Anatomía Veterinaria de la Universidad Santo Tomás, (UST) sede Puerto Montt, Chile.

La descripción anatómica aborda a las estructuras en estudio desde una vista general hasta una descomposición de tal estructura, con el fin de orientar al lector espacial y topográficamente así como en su forma general y apariencia, para luego caracterizar detenidamente cada uno de los segmentos y piezas que componen ese todo. 
En consecuencia a lo anterior, el método de estudio que se utilizó es de tipo descriptivo cualitativo. En el que se realizó la observación y descripción de piezas óseas (cráneo).

El desarrollo descriptivo siguió un orden metódico; donde los conceptos utilizados para referirse a las diferentes estructuras (vista y planos de observación, topografía, disposición espacial, relaciones subyacentes y nombres) fueron tomados de la nómina anatómica veterinaria (International Committee on Veterinary Gross Anatomical Nomenclature); dando inicio con una vista general en los distintos planos del esqueleto craneal, para continuar con una división esquemática de los dos segmentos principales que componen el cráneo (neurocráneo y esplacnocráneo) y de cada uno de los huesos que componen estos segmentos óseos.

Los resultados obtenidos de la descripción anatómica del cráneo, fueron analizados y discutidos bajo criterios de ausencia o presencia de estructuras con respecto a lo descrito en la bibliografía anatómica para pequeños rumiantes domésticos (como el Caprino o el Ovino). Esto se fundamenta en el hecho de que existe relación taxonómica y filogénica entre el Pudú y este grupo de especies, categorizándolos a lo menos como rumiantes de pequeña talla, como menciona, Guzmán (2007) en su estudio genético del Pudú, donde describe que las familias Bovidae y Cervidae presentan cierto grado de parentesco.

El objetivo de este trabajo no es más que describir la osteología craneana de la especie Pudu puda y la comparación con otros rumiantes de pequeña talla, se basa en el hecho de hacer aún más concluyentes los resultados y de entregar al lector una referencia de la arquitectura ósea de los pequeños rumiantes domésticos.

Las piezas óseas que se utilizaron en el desarrollo de este trabajo pertenecen a la unidad de anatomía veterinaria de la Universidad Santo Tomás sede Puerto Montt y fueron obtenidas años previos a este estudio a través de técnica de osteotecnia (Rodríguez Palomo \& Ramírez Zamora, 2009). Esta técnica fue realizada en cadáveres de Pudú conservados, ambos obtenidos por donación de funcionarios del SAG Puerto Montt con fines pedagógicos.

Para la observación en profundidad de los distintos segmentos del cráneo del Pudú, se realizó un corte en el plano sagital mediano del esqueleto craneal y un corte en el plano dorsal del neurocráneo. Esto permitió tener una apreciación de la arquitectura del cráneo tanto de una vista externa general como de una vista interna de la cavidad nasal y cavidad endocraneana.

\section{RESULTADOS Y DISCUSIÓN}

Esqueleto Craneal. El cráneo o esqueleto craneal de la especie Pudu puda, al igual que en todos los vertebrados, está conformado por dos segmentos principales, neurocráneo y esplacnocráneo. Ya desde la etapa embrionaria hay una clara diferenciación entre ambos segmentos futuramente óseos y en mamíferos en estado adulto generalmente se manifiesta con un mayor y amplio desarrollo de los huesos faciales (esplacnocráneo) frente a los huesos de la parte neural (neurocráneo) similitud a lo planteado en publicaciones de anatomía de rumiantes (Gloobe, 1989; Shively, 1993; Popesko, 1998; Sisson \& Grossman, 1999; Budras \& Habel, 2003; Köning \& Liebich, 2005; Dyce et al., 2012) (Fig. 3).

Al realizar una apreciación general del esqueleto craneal del Pudú, se observa más estilizado, largo y homogéneo en relación a rumiantes domésticos de pequeña talla (caprino y ovino), dado esto posiblemente por el mayor ángulo con el que se relacionan el neurocráneo y esplacnocráneo, por la prolongación del hueso frontal y parietal en la formación del techo del neurocráneo, así como por la disminución en el eje dorso-ventral de los huesos maxilares en la formación de la cara. Además, la menor proyección lateral de los márgenes de la órbita ocular, le otorgan ese aspecto homogéneo y estilizado al esplacnocráneo, esto último determina posiblemente, el enfoque más frontal de los globos oculares característicos de la especie (Fig. 1).

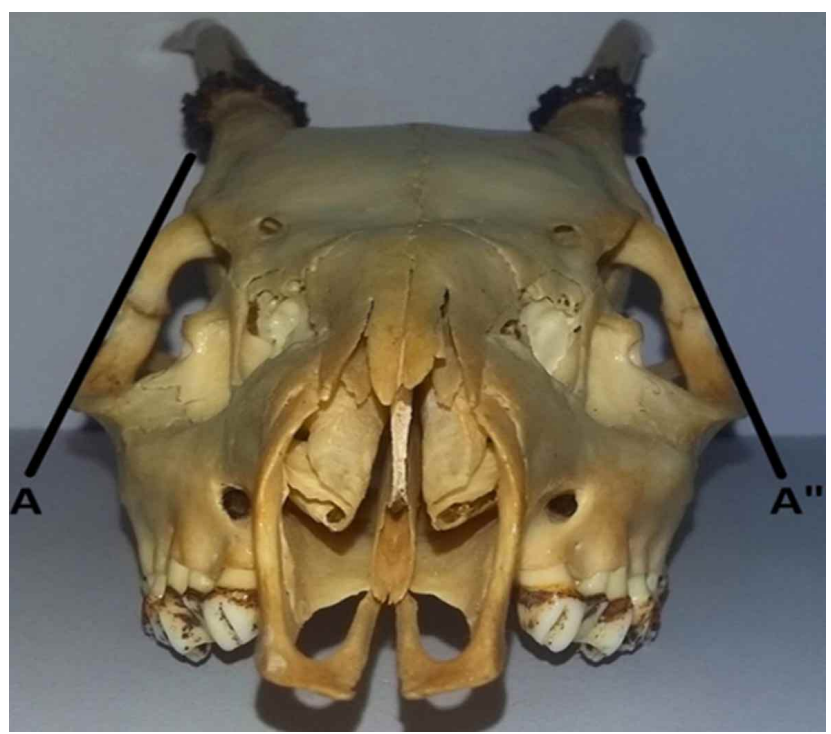

Fig. 1. Vista rostral del esqueleto craneal de un ejemplar macho de la especie Pudu puda en la que se muestran los limites laterales de esplacnocráneo, dados por el margen lateral del hueso cigomático. Fuente: unidad de anatomía veterinaria, UST, sede Puerto Montt, Chile. 


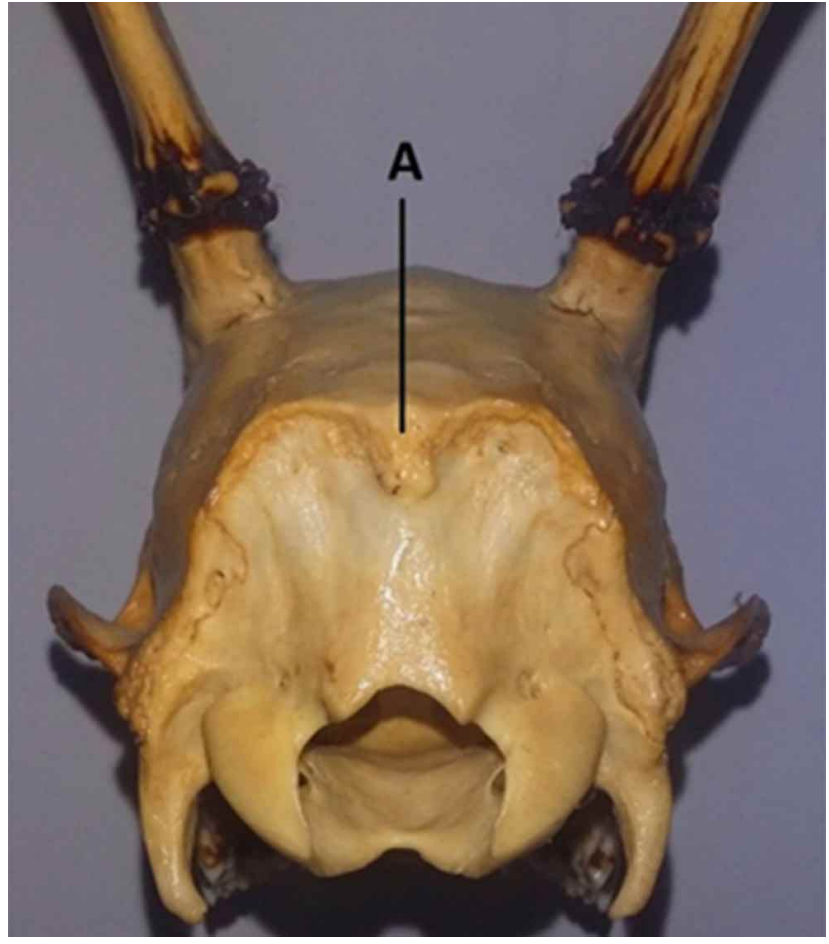

Fig. 2. Vista caudal del esqueleto craneal de un ejemplar macho de la especie $P u d u$ puda en la que destaca la protuberancia occipital externa (A). Fuente: Unidad de anatomía veterinaria, UST, sede Puerto Montt, Chile.

\section{Neurocráneo}

Hueso Occipital. En el hueso occipital del Pudú se observan tres porciones, basilar, lateral y escamosa. En esta última se observa la protuberancia occipital externa formando en su totalidad el margen dorsal de la pared caudal de la cavidad craneana, ésta destaca por ser bastante definida, se proyecta lateralmente donde a su paso articula con el margen caudal del hueso temporal en su porción petrosa internamente y timpánica externamente. Mientras que en la porción basilar destacan los tubérculos musculares, que se encuentran más cercanos hacia la línea sagital media, siendo el extremo rostral de ésta porción más angosta y así dando forma triangular a la base del neurocráneo.

\section{Hueso Esfenoides}

Basisfenoides. Debido a la cercanía de los tubérculos musculares hacia el ángulo sagital medio en la porción basilar del occipital, el cuerpo del basisfenoides mantiene esta congruencia en su forma y adopta también una forma triangular y más angosta, a diferencia de lo que ocurre en el caprino y ovino, donde la apariencia es rectangular. En una vista endocraneana del cuerpo del basisfenoides, se observa la fosa hipofisiaria y el dorso selar junto a sus procesos clinoideos como límite caudal (Fig. 4).

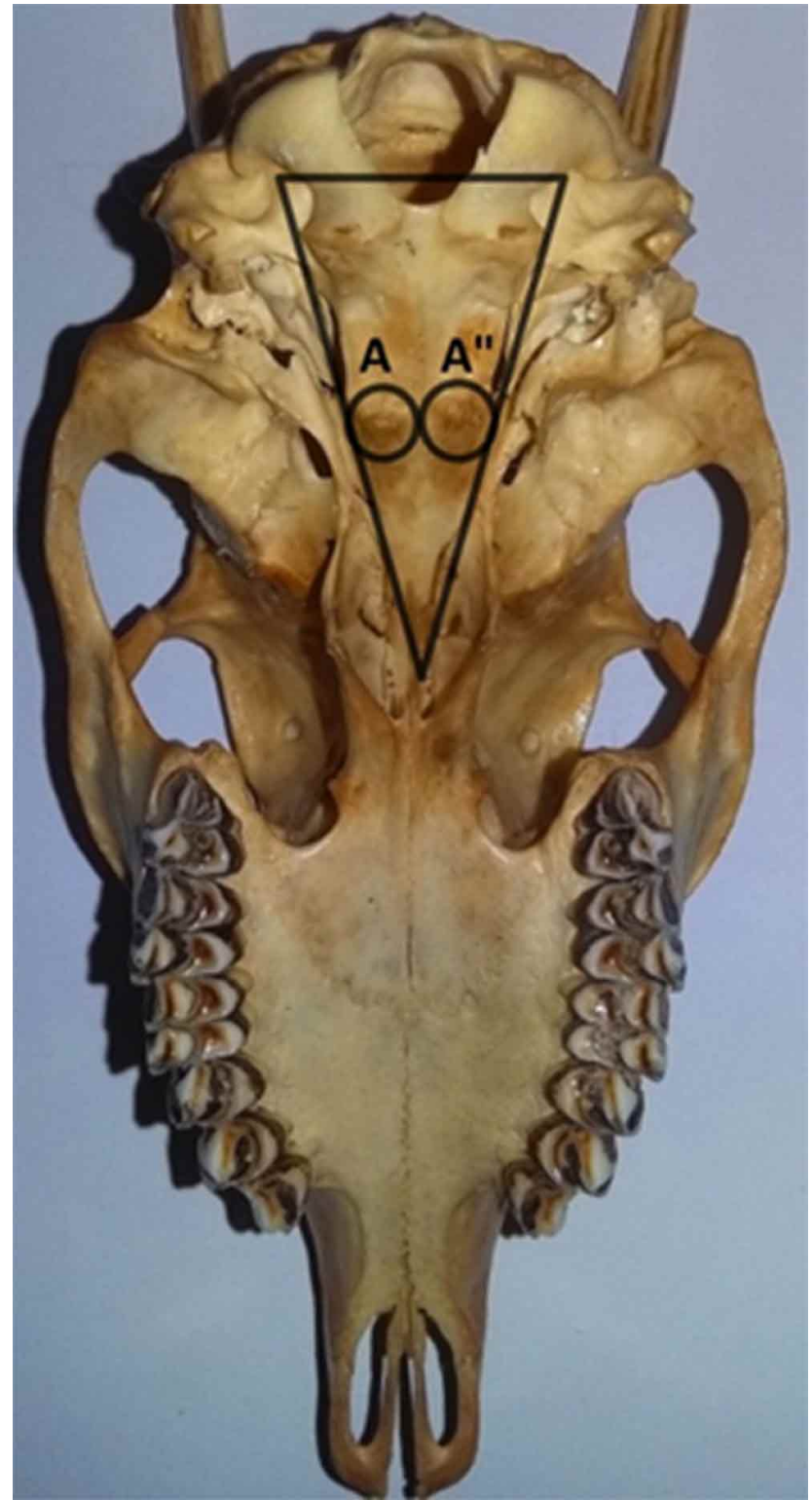

Fig. 3. Vista ventral del esqueleto craneal de un ejemplar macho de la especie Pudu puda en la que destacan los procesos musculares de la porción basilar del hueso occipital (A y A"). Fuente: Unidad de anatomía veterinaria, UST, sede Puerto Montt, Chile.

Presfenoides. El hueso presfenoides continúa rostralmente desde el basisfenoides formando la base del cráneo. En una vista interna y después de la fosa hipofisaria formada por el cuerpo del basisfenoides, se observan sus alas levantando el piso y así estrechando la cavidad craneana, para luego articular con la porción orbital del hueso frontal (Fig. 5).

Hueso Temporal. Al igual que en otros pequeños rumiantes domésticos, el hueso temporal del Pudú participa en la formación de las paredes laterales de la cavidad craneana. En la observación destaca la perdida de continuidad entre 


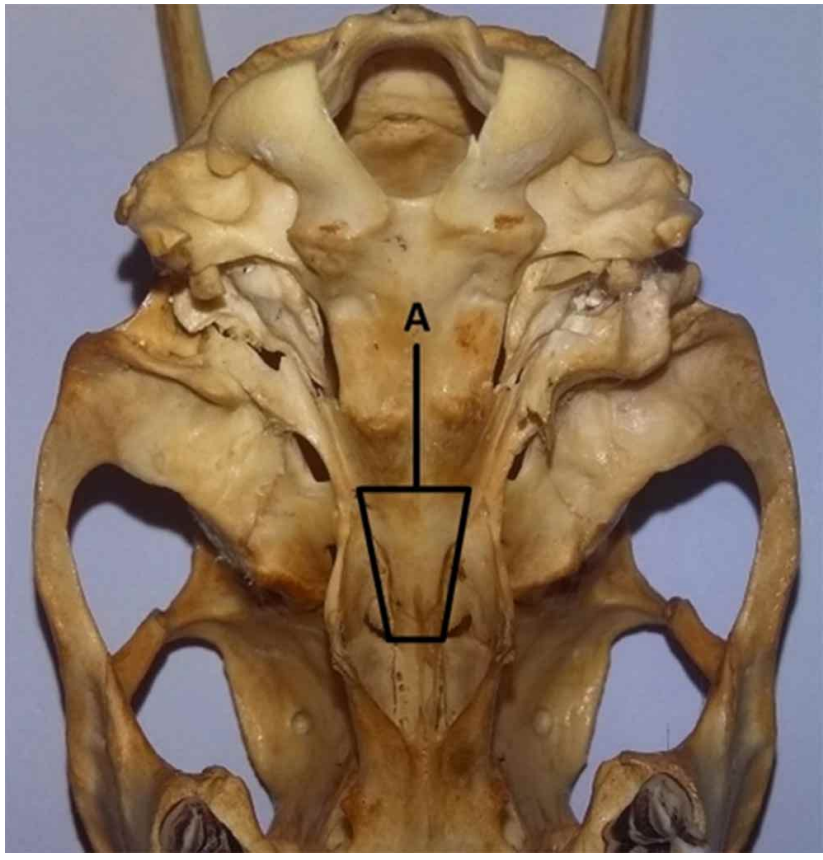

Fig. 4.Vista ventral del esqueleto craneal de un ejemplar macho de la especie Pudu puda en la que destaca el hueso basisfenoides. Fuente: Unidad de anatomía veterinaria, UST, sede Puerto Montt, Chile.

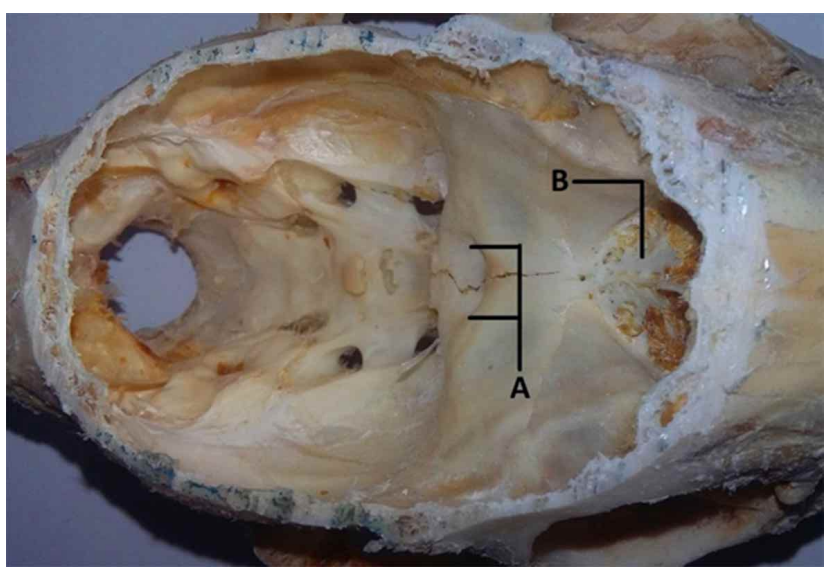

Fig. 5.Vista dorsal de la cavidad endocraneana de un ejemplar hembra de la especie Pudu puda en la que se destacan: las alas del presfenoides (A), hueso etmoides (B). Fuente: unidad de anatomía veterinaria, UST, sede Puerto Montt, Chile.

los márgenes laterales de la pared caudal de la cavidad craneana y el nacimiento del proceso cigomático desde la porción escamosa del temporal, dado esto por la proyección dorso lateral del meato acústico externo. Situación diferente a lo ocurrido en el ovino y caprino, donde el meato acústico externo se proyecta lateralmente ventral al nacimiento del proceso cigomático (Fig. 6). Guardando similitud por lo planteado en las publicaciones en animales rumiantes (Sisson \& Grossman; Gloobe; Shively; Köning $\&$ Liebich).

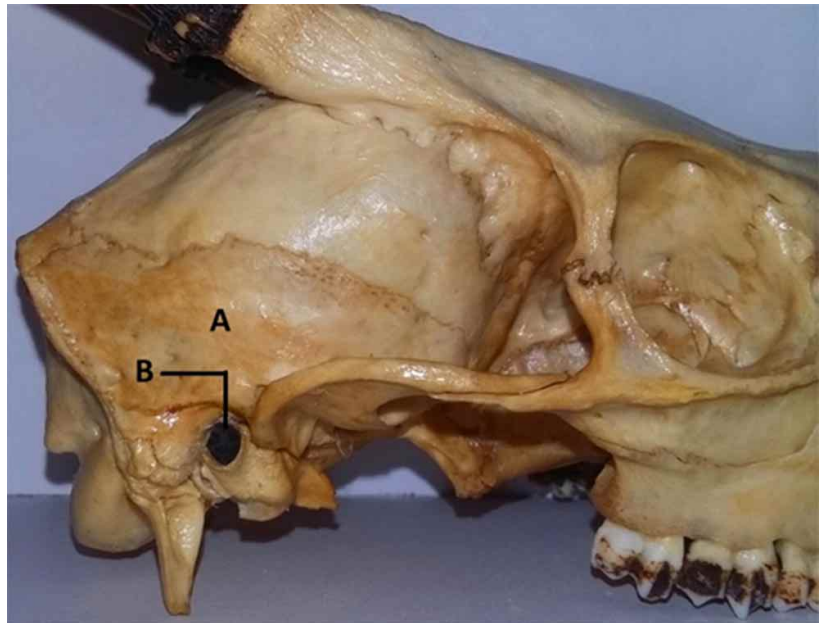

Fig. 6. Vista lateral izquierda de esqueleto craneal de un ejemplar macho de la especie Pudu puda en la que se destacan: Hueso temporal (A), meato acústico externo (B). Fuente: unidad de anatomía veterinaria, UST, Puerto Montt, Chile.

Hueso Parietal. En el Pudú, el hueso parietal alcanza una gran extensión, formando gran parte del techo y de las paredes laterales de la cavidad craneana. La sutura a través de la cual articula en su margen rostral con el hueso frontal, marca el nacimiento en proyección caudal de los procesos cornuales del hueso frontal, situación similar a la que ocurre en el chivo pero muy diferente en la oveja.

Hueso Frontal. Es un hueso extenso que forma la mitad rostral del techo de la cavidad craneana, la otra mitad la completa el hueso parietal. Lateralmente en la porción escamosa, se proyecta el proceso cigomático que participa en la formación del margen dorso caudal de la órbita ocular, que a diferencia de otros pequeños rumiantes domésticos, este margen no sobresale de los límites laterales de la cara, dando así una apariencia más aguzada al esqueleto craneal. Aquí también se encuentra el agujero supraorbital.

Desde el margen caudal de este hueso se proyectan en dirección caudal los procesos cornuales, presentes solo en el macho. En el nacimiento de los procesos cornuales destaca una depresión o fosa, que coincide también con el nacimiento del proceso cigomático del hueso frontal (Fig. 7).

Los huesos etmoidales, aparato hioideo e interparietal, con partes similitudes en su arquitectura en relación a pequeños rumiantes domésticos.

\section{Esplacnocráneo}

Hueso Maxilar. El hueso maxilar presenta una forma alargada en su eje rostro-caudal e irregular y angosta en su eje 


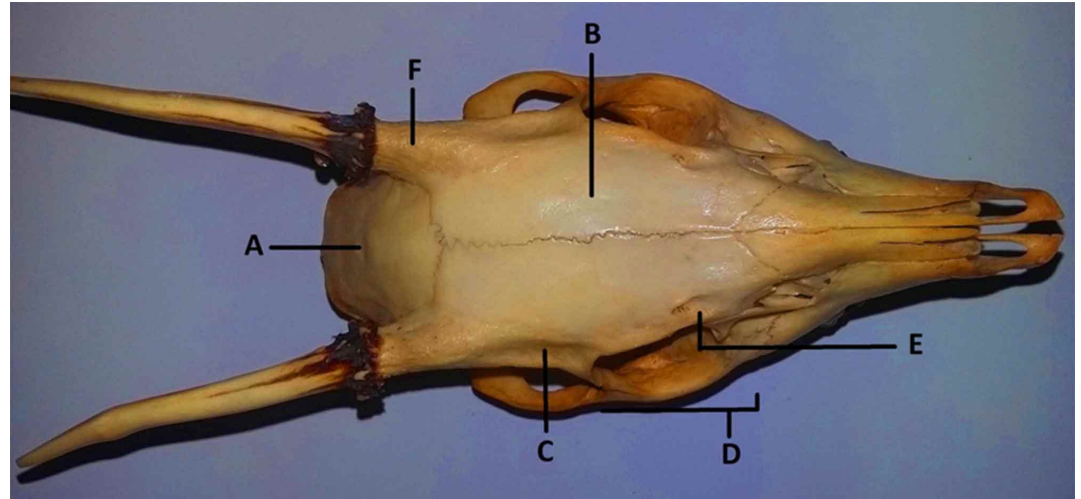

Fig. 7. Vista dorsal del esqueleto craneal de un ejemplar macho de la especie Pudu puda en la que se observan: Hueso parietal (A), Hueso frontal (B), Fosa en el nacimiento de los procesos cornuales y procesos cigomáticos del hueso frontal (C), Margen lateral de la cara marcado por el hueso cigomático (D), Agujero supraorbital (E), Procesos cornuales (F). Fuente: Unidad de anatomía veterinaria, UST, sede Puerto Montt, Chile.

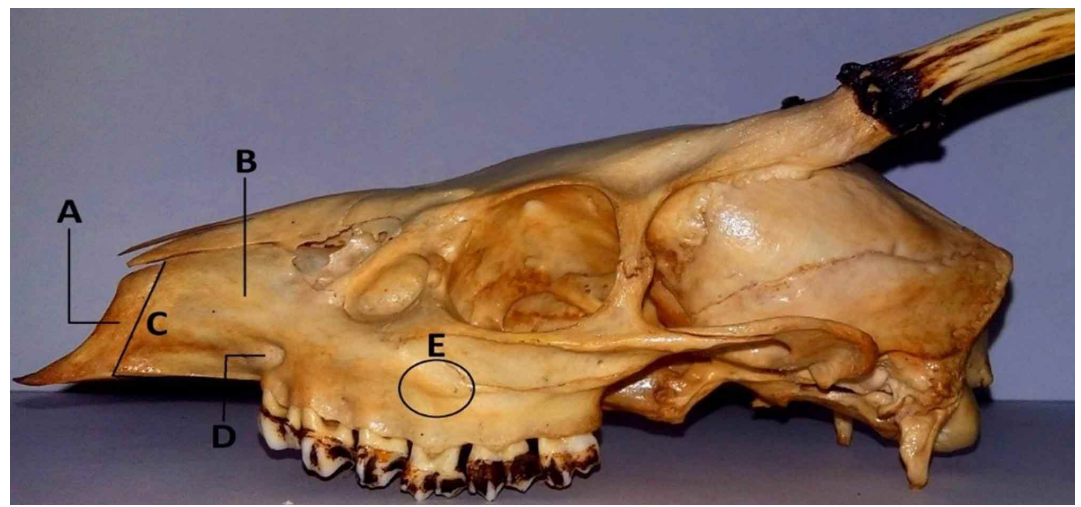

Fig. 8. Vista lateral izquierda del esqueleto craneal de un ejemplar macho de la especie Pudu puda en la que se destacan: Hueso incisivo (A), Hueso Maxilar (B), Ángulo de la articulación entre el H. palatino y H. maxilar, Agujero infraorbitario (D), Tubérculo facial (E). Fuente: Unidad de anatomía veterinaria, UST, sede Puerto Montt, Chile.

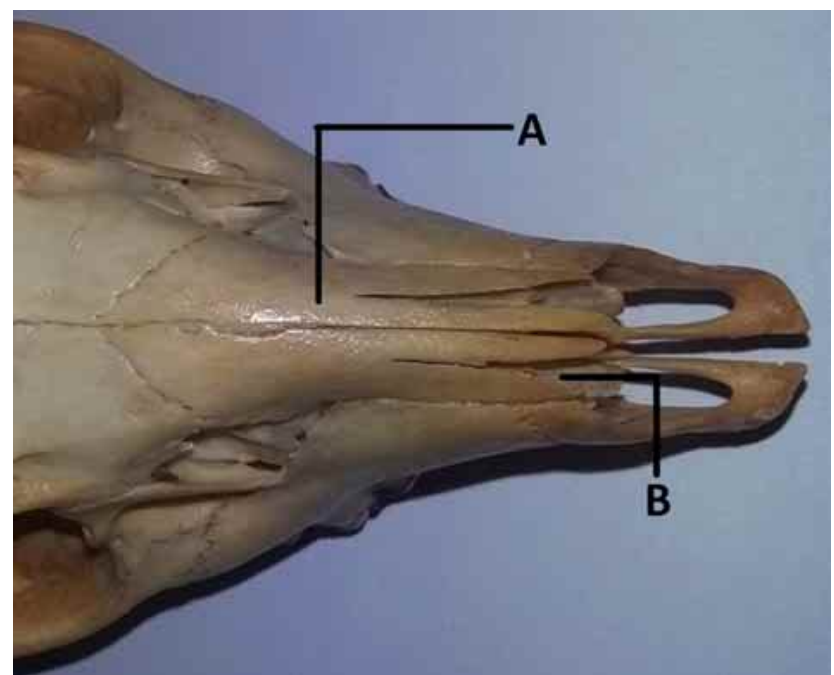

Fig. 9. Vista dorsal del esqueleto craneal de un ejemplar macho de la especie Pudu puda en la que se destacan: hueso nasal (A), incisura propia del hueso nasal (B). Fuente: unidad de anatomía veterinaria, UST, sede Puerto Montt, Chile. dorso-ventral, en esto influye en parte, la presencia de un gran hueso lagrimal y su amplia fosa externa, no obstante, su margen rostral posee un menor ángulo al articular con el hueso incisivo, lo que da una apariencia menos aguzada al comparar con el esplacnocráneo de otros pequeños rumiantes de pequeña talla.

En la superficie externa se observa un tubérculo facial menos desarrollado y menos prominente que en otros pequeños rumiantes, ubicándose incluso bajo la órbita ocular y hueso cigomático. Mientras que el agujero infraorbitario se encuentra en disposición rostral sobre el primer premolar.

En cuanto a la dentadura, en la arcada superior se observan: 6 piezas, $2 \mathrm{x}$ (3 premolares y 3 molares); Sin incisivos superiores (Fig. 8).

Hueso Incisivo. El hueso incisivo presenta un ángulo menor con respecto a la articulación con el hueso maxilar en su extremo rostral, a diferencia de lo que ocurre en otros pequeños rumiantes domésticos.

Hueso Nasal. Al igual que en pequeños rumiantes domésticos, el hueso nasal es un hueso par y forma el techo de la cavidad nasal, en el Pudú destaca por ser aguzado en su extremo rostral, presentar incisura nasoincisiva y además por estar dividido en su eje mayor hasta la mitad rostral por una incisura propia del hueso (Fig. 9).

Hueso Palatino. En cuanto a la formación del paladar duro y la articulación con el proceso palatino del maxilar a través de la lámina horizontal, no se manifiestan diferencias entre el Pudú y otros pequeños rumiantes domésticos (Sisson \& Grossman; Gloobe; Ashdown \& Done, 2011). Sin embargo destacan; la presencia de un par de procesos ubicados en la lámina perpendicular, en el nacimiento del meato nasofaríngeo y las coanas, y el menor diámetro de la lámina perpendicular y por ende la menor profundidad del meato nasofaríngeo. Esto último dado posiblemente por la posición y ángulo del neurocráneo con respecto al esplacnocráneo, donde en una vista general del cráneo se aprecia un mayor ángulo y por lo tanto un esqueleto craneal más estilizado. 


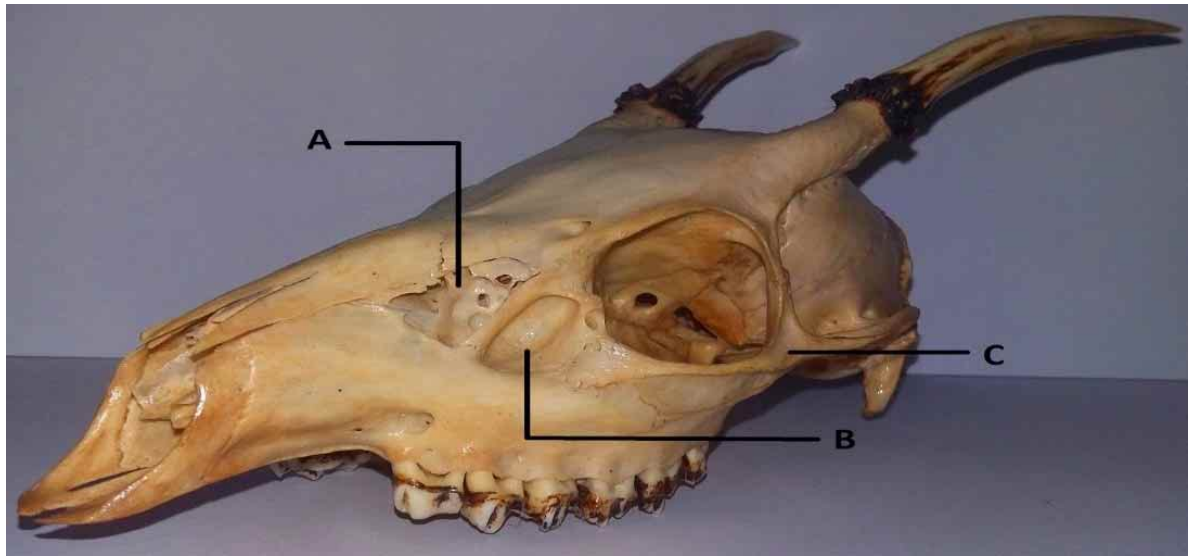

Fig. 10. Vista oblicua rostro lateral del esqueleto craneal de un ejemplar macho de la especie $P u d u$ puda, en la que se destacan: Abertura entre los huesos frontal, nasal, maxilar y lagrimal, asociada a la funcionalidad del sistema lagrimal (A), fosa lagrimal externa del hueso lagrimal (B), Hueso cigomático (C). Fuente: unidad de anatomía veterinaria, UST, sede Puerto Montt., Chile. lagrimal y bajo ella el inicio del conducto nasolagrimal. Mientas que en la superficie facial se observa una amplia fosa lagrimal externa, ésta destaca por tener gran profundidad. Entre el hueso lagrimal, maxilar, nasal y frontal, se genera una abertura horadada que comunica con los senos paranasales y cavidad nasal, descrita solo en el caprino (Fig. 10).

Hueso Cigomático. Se presenta articulado desde su extremo rostral al hueso maxilar, con proyección caudal donde articula con el hueso frontal y cigomático. Destaca por carecer de proyección lateral, siendo más delgado que aplanado a diferencia de lo que ocurre en otros pequeños rumiantes domésticos (Fig. 10).

Mandíbula. La mandíbula se conforma de dos segmentos, lateral derecho y lateral izquierdo, cada uno presenta un cuerpo y una rama (Sisson \& Grossman; Gloobe; Shively). Destaca en la rama, un proceso angular prominente y acentuado, dado por la presencia de una marcada escotadura en el límite con el cuerpo por el margen ventral, con mayor desarrollo en machos que en hembras. Por su parte el proceso coronoideo destaca por su gran proyección caudal, siendo también mayor en machos. En cuanto a la dentadura, se observa la presencia de 2x (4 incisivos, 1 canino, 2 premolares, 3 molares) (Figs. 11 y 12$)$.

\section{CONCLUSIONES}

Las observaciones realizadas al esqueleto craneal de la especie Pudu puda y la posterior descripción de los segmentos óseos que lo componen, determinan que existen diferencias con respecto al cráneo de pequeños rumiantes domésticos.

Se logró enfatizar las principales diferencias existentes a nivel de neurocráneo y esplacnocráneo, presentando extensiones, depresiones y fosas que destacan la arquitectura del cráneo de esta especie.

Es necesario considerar que la descripción anatómica no es suficiente para establecer relaciones o parámetros cuantitativos a nivel del cráneo de esta especie, es por ello que la continuidad de este estudio se profundizara en dimensionar demanera morfométrica, cada diferencia observada. 
SALDIVIA, M. \& VILLEGAS, F. Anatomical description of the bone segments that make up the skull of the Pudu puda species. Int. J. Morphol., 37(1):167-173, 2019.

SUMMARY: The Pudú (Pudu puda), one of the smallest deer in the world and indigenous to Chile, is considered vulnerable (VU) throughout the national territory, there is little specific information on the organic systems that compose it, and it is becoming more common find them in urban areas. These problems awaken the need to strengthen research and study of this species. Consequently, in the present study an anatomical description of the cranial skeleton of two specimens of Pudú puda was made, where their main bony characteristics were highlighted by comparing them with domestic species already studied (sheep and goats). Thus, it was possible to determine differences between the species studied and the need to deepen the analysis through measurements of the bone pieces.

KEY WORDS: Pudu; Anatomical description; Cranial skeleton.

\section{REFERENCIAS BIBLIOGRAFICAS}

Ashdown, R. R. \& Done, S. H. Atlas en Color de Anatomía Veterinaria. Rumiantes. $2^{\mathrm{a}}$ ed. Madrid, Elsevier, 2011.

Budras, K. D. \& Habel, R. E. Bovine Anatomy. An Illustrated Text. Hannover, Schlütersche, 2003.

Chester, S. A Wildlife Guide to Chile. Barcelona, Lynx, 2008.

Dyce, K. M; Sack, W. O. \& Wensing, C. J. G. Anatomía Veterinaria. $4^{\mathrm{a}}$ ed. Ciudad de México, El Manual Moderno, 2012.

Gloobe, H. Anatomía Aplicada del Bovino. San José, Instituto Interamericano de Cooperación para la Agricultura (IICA), 1989.

Guzmán, R. B. E. Evaluación de Loci Microsatelitales Heterólogos y Utilización de Secuencias de Citocromo Oxidasa I Mitocondrial en Estudios Genéticos de Pudú Puda (Artiodactyla, Cervidae). Tesis de Grado, Licenciatura en Ciencias Biológicas. Valdivia, Universidad Austral de Chile, 2007.

International Committee on Veterinary Gross Anatomical Nomenclature. Nomina Anatomica Veterinaria. $5^{\text {a }}$ ed. Hannover, Editorial Committee, 2012.

Köning, H. \& Liebich, H. Anatomía de los Animales Domésticos. $2^{\mathrm{a}}$ ed. Madrid, Médica Panamericana, 2005.

Popesko, P. Atlas de Anatomía Topográfica de los Animales Domésticos. $2^{\mathrm{a}}$ ed. Barcelona, Masson, 1998.

Rodríguez Palomo, D. \& Ramírez Zamora, J. Técnica de conservación de huesos en peróxido de hidrógeno. Med. Leg. Costa Rica, 26(2):11723, 2009.

Shively, M. Anatomía Veterinaria Básica, Comparada y Clínica. Ciudad de México, El Manual Moderno, 1993.

Sisson, S. \& Grossman, J. Anatomía de los Animales Domésticos. 5a ed. Barcelona, Masson, 1999.

\author{
Dirección para correspondencia: \\ Manuel Saldivia P. \\ Medico Veterinario \\ Tec. en Veterinaria y Produccion Pecuaria \\ Santo Tomas -CFT \\ Puerto Montt \\ CHILE
}

Email: vetmanuelch@hotmail.com

Recibido : 30-05-2018

Acpetado: 29-10-2018 